\title{
A POLYHEDRAL STUDY OF A TWO LEVEL FACILITY LOCATION MODEL *
}

\author{
Mourad Bä̈OU ${ }^{1}$ And Francisco BARAhona ${ }^{2}$
}

\begin{abstract}
We study an uncapacitated facility location model where customers are served by facilities of level one, then each level one facility that is opened must be assigned to an opened facility of level two. We identify a polynomially solvable case, and study some valid inequalities and facets of the associated polytope.
\end{abstract}

Keywords. Uncapacitated facility location problem, two level facility location.

Mathematics Subject Classification. 05C85, 90C27.

\section{INTRODUCTION}

We study a two level uncapacitated facility location model. Each customer has to be assigned to an opened facility of the first level, then each opened facility of the first level must be assigned to an opened facility of the second level. There are fixed costs for each assignment, and fixed costs for opening each facility. The objective is to minimize the total cost. This represents a hierarchical structure with minor regional depots and major central warehouses. Here we study a polytope associated with this model, and identify a polynomially solvable case. Surprisingly the only reference to this model that we found is [10] where an approximation algorithm has been given for the extension to $k$ levels.

Received September 11, 2013. Accepted November 28, 2013.

* This work is supported by the project PICS05891 CNRS and IBM.

1 CNRS, LIMOS, and Université Blaise Pascal, Complexe scientifique des Cézeaux, 63173

Aubiere Cedex, France.

mourad.baiou@isima.fr

2 IBM T. J. Watson research Center, Yorktown Heights, NY 10589, USA.

barahon@us.ibm.com 
A different related model arises when each client must be serviced by a sequence of $k$ different facilities, each of these sequences has a transportation cost, and each facility has a fixed cost. An approximation algorithm for this case has been given in [1]. A polytope associated with the two level version of this last model has been studied in [2].

Now we give a precise definition of the problem. Let $G=(V, A)$ be a tripartite directed graph, with $V=V_{0} \cup V_{1} \cup V_{2}, V_{i} \cap V_{j}=\emptyset$, for $i \neq j ; A=A_{1} \cup A_{2}$, $A_{1} \subseteq V_{0} \times V_{1}$ and $A_{2} \subseteq V_{1} \times V_{2}$. Here $V_{0}$ corresponds to a set of customers, $V_{1}$ corresponds to a set of potential facilities of level one, and $V_{2}$ corresponds to a set of potential facilities of level two. An integer programming formulation of the two level facility location problem is

$$
\begin{aligned}
& \min \sum_{(u, v) \in A_{1} \cup A_{2}} c(u, v) x(u, v)+\sum_{v \in V_{1} \cup V_{2}} f(v) y(v) \\
& \sum_{(u, v) \in A_{1}} x(u, v)=1, \quad \forall u \in V_{0} \\
& x(u, v) \leq y(v), \quad \forall(u, v) \in A_{1} \\
& \sum_{(u, v) \in A_{2}} x(u, v)=y(u), \quad \forall u \in V_{1} \\
& x(u, v) \leq y(v), \quad \forall(u, v) \in A_{2} \\
& x(u, v) \in\{0,1\}, \quad \forall(u, v) \in A_{1} \cup A_{2} \\
& y(v) \in\{0,1\}, \quad \forall v \in V_{1} \cup V_{2} .
\end{aligned}
$$

The variable $x(u, v)$ represents the assignment from $u$ to $v$, the cost of this assignment is $c(u, v)$. The cost of opening a facility $v$ is $f(v)$. Equation (1.1) imply that each customer should be assigned to one facility of level one. Equation (1.3) imply that if a facility of level one is opened, then it should be assigned to a facility of level two. Inequalities (1.2) and (1.4) imply that if a facility is not opened then no assignment can be made to that facility.

We call two level facility location polytope of $G(T L F L P(G))$ the convex hull of all vectors satisfying $(1.1)-(1.6)$. Let $P(G)$ be the polytope defined by $(1.1)-(1.4)$ and

$$
\begin{aligned}
& 0 \leq x(u, v) \leq 1, \quad \forall(u, v) \in A_{1} \cup A_{2} \\
& 0 \leq y(v) \leq 1, \quad \forall v \in V_{1} \cup V_{2} .
\end{aligned}
$$

Clearly $T L F L P(G) \subseteq P(G)$, in this paper we characterize the graphs for which TLFLP $(G)=P(G)$. For this class of graphs the problem is polynomially solvable. We also study the facial structure of $T L F L P(G)$.

This paper is organized as follows. In Section 2 we give some definitions. In Section 3 we give some inequalities that are valid for $T L F L P(G)$. In Section 4 we characterize the graphs for which $P(G)=T L F L P(G)$. Section 5 is devoted to study the facial structure of $\operatorname{TLFLP}(G)$. 


\section{Preliminaries}

In this section we give some definitions and notations. Given a graph $G=(V, A)$, a simple cycle $C$ is an ordered sequence $v_{0}, a_{0}, v_{1}, a_{1}, \ldots, a_{t-1}, v_{t}=v_{0}$, where all $v_{i}$ 's, for $i=0, \ldots, t-1$, are distinct and such that for $0 \leq i \leq t-1$ : either $v_{i}$ is the tail of $a_{i}$ and $v_{i+1}$ is the head of $a_{i}$, or $v_{i}$ is the head of $a_{i}$ and $v_{i+1}$ is the tail of $a_{i}$. Let $V(C)$ and $A(C)$ denote the nodes and the arcs of $C$, respectively. By setting $a_{t}=a_{0}$, we associate with $C$ three more sets as below.

- We denote by $\hat{C}$ the set of nodes $v_{i}$, such that $v_{i}$ is the head of $a_{i-1}$ and also the head of $a_{i}, 1 \leq i \leq t$.

- We denote by $\dot{C}$ the set of nodes $v_{i}$, such that $v_{i}$ is the tail of $a_{i-1}$ and also the tail of $a_{i}, 1 \leq i \leq t$.

- We denote by $\tilde{C}$ the set of nodes $v_{i}$, such that either $v_{i}$ is the head of $a_{i-1}$ and also the tail of $a_{i}$, or $v_{i}$ is the tail of $a_{i-1}$ and also the head of $a_{i}, 1 \leq i \leq t$.

Notice that $|\hat{C}|=|\dot{C}|$. A cycle will be called $g$-odd if $|\tilde{C}|+|\hat{C}|$ is odd, otherwise it will be called g-even. A cycle $C$ with $V(C)=\tilde{C}$ is a directed cycle. The notion of $g$-odd ( $g$-even) cycles generalizes the notion of odd (even) directed cycles. Also a cycle $C$ is called $d$-odd ( $d$-even) if $|\dot{C}|$ is odd (even).

A polyhedron is called integral if all its extreme points are integral. A polytope is a bounded polyhedron. An inequality $a x \leq \alpha$ is called valid with respect to a polyhedron $P$, if $P \subseteq\{x \mid a x \leq \alpha\}$. Valid inequalities are useful if they cut off factional extreme points of a linear programming relaxation of an integer programming problem. If $a x \leq \alpha$ is valid for a polyhedron $P$, then $F=\{x \in P \mid a x=\alpha\}$ is called a face of $P$. A facet of $P$ is a maximal face of $P$. If an inequality defines a facet, it can be viewed as a "strongest" valid inequality.

Given a vector $x \in \mathbb{R}^{E}$, we denote by $x(S)$ the sum $\sum_{e \in S} x(e)$. We denote by $G_{1}$ the subgraph of $G$ induced by $V_{0} \cup V_{1}$. We also denote by $G_{2}$ the subgraph of $G$ induced by $V_{1} \cup V_{2}$. For a directed graph $G=(V, A)$ and a set $W \subset V$, we denote by $\delta^{+}(W)$ the set of $\operatorname{arcs}(u, v) \in A$, with $u \in W$ and $v \in V \backslash W$. We write $\delta^{+}(v)$ instead of $\delta^{+}(\{v\})$.

For a directed graph $H=(W, B)$, we call the (one level) facility location polytope of $H(F L P(H))$ the convex hull of all vectors satisfying

$$
\begin{aligned}
& \sum_{(u, v) \in A} x(u, v)+y(u) \leq 1 \quad \forall u \in W, \\
& x(u, v) \leq y(v) \quad \forall(u, v) \in B, \\
& y(v) \in\{0,1\} \quad \forall v \in W, \\
& x(u, v) \in\{0,1\} \quad \forall(u, v) \in B .
\end{aligned}
$$

This polytope was studied in [4], and a class of valid inequalities was given as follows. If $C$ is a $g$-odd cycle of $H$, then the inequality below is valid for $F L P(H)$.

$$
\sum_{a \in A(C)} x(a)-\sum_{v \in \hat{C}} y(v) \leq \frac{|\tilde{C}|+|\hat{C}|-1}{2} .
$$


For a family of inequalities, the separation problem consists of given a vector $(\bar{x}, \bar{y})$, finding an inequality in this family that is violated by $(\bar{x}, \bar{y})$, if there is any. An algorithm for the separation of inequality (2.1) was given in [4], its complexity is $O\left(|W|^{2}|B|^{2}\right)$. An extension of these inequalities to the two level case will be studied in the next section.

For an undirected graph $U=(W, E)$, the stable set polytope is the convex hull of all vectors satisfying

$$
\begin{aligned}
& x(i)+x(j) \leq 1 \text { for every edge }\{i, j\} \in E, \\
& x(i) \in\{0,1\} \text { for every node } i \in V .
\end{aligned}
$$

\section{VALID INEQUALITIES}

In this section we present three families of valid inequalities for $T L F L P(G)$ and discuss their separation problem.

\section{1. $d$-ODD CYCLE INEQUALITIES}

Lemma 3.1. If $C$ is a d-odd cycle, then the following inequality is valid for $T L F L P(G)$.

$$
\sum_{u \in \dot{C},(u, v) \in A(C)} x(u, v)-\sum_{v \in \hat{C}} y(v)-\sum_{v \in \tilde{C}} x\left(\delta^{+}(v) \backslash A(C)\right) \leq \frac{|\dot{C}|-1}{2} .
$$

Proof. From inequalities (1.1)-(1.4) and (1.7)-(1.8) we obtain

$$
\begin{aligned}
& x(u, v)-y(v) \leq 0, \text { for every } \operatorname{arc}(u, v) \in A(C), v \in \tilde{C} \cup \hat{C}, \\
& y(v)-x\left(\delta^{+}(v)\right)=0, v \in \tilde{C}, \\
& x\left(\delta^{+}(v)\right) \leq 1, \text { for } v \in \dot{C}, \\
& -x(u, v) \leq 0, \text { for }(u, v) \in \delta^{+}(u) \backslash A(C), \text { for } u \in \dot{C} \cup \tilde{C} .
\end{aligned}
$$

Their sum gives

$$
2 \sum_{u \in \dot{C},(u, v) \in A(C)} x(u, v)-2 \sum_{v \in \hat{C}} y(v)-2 \sum_{v \in \tilde{C}} x\left(\delta^{+}(v) \backslash A(C)\right) \leq|\dot{C}| .
$$

Dividing by 2 and rounding down the right hand side, as in Chvátal's procedure [8], we obtain inequality (3.1).

In the next section we show fractional vectors in $P(G)$ that are separated from TLFLP $(G)$ by inequality (3.1). Now we discuss how to solve the separation problem for inequality (3.1). Assume that $(\bar{x}, \bar{y})$ is a vector of $P(G)$. We first build a graph $H=(W, B)$, where $W=V_{0} \cup V_{1}^{\prime} \cup V_{2}$, and

$$
\begin{aligned}
& V_{1}^{\prime}=\left\{v^{\prime} \mid v \in V_{1}\right\} \cup\left\{v^{\prime \prime} \mid v \in V_{1}\right\}, \\
& B=\left\{\left(u, v^{\prime}\right) \mid(u, v) \in A_{1}\right\} \cup\left\{\left(v^{\prime \prime}, w\right) \mid(v, w) \in A_{2}\right\} \cup\left\{\left(v^{\prime}, v^{\prime \prime}\right) \mid v \in V_{1}\right\} .
\end{aligned}
$$


So the graph $H$ is obtained by splitting every node in $V_{1}$ into two copies, and adding an arc from the first copy to the second copy. Now we build a vector $\left(x^{\prime}, y^{\prime}\right)$ as follows,

$$
\begin{aligned}
x^{\prime}\left(u, v^{\prime}\right) & =\bar{x}(u, v), \quad \forall(u, v) \in A_{1}, \\
x^{\prime}\left(v^{\prime \prime}, w\right) & =\bar{x}(v, w), \quad \forall(v, w) \in A_{2}, \\
x^{\prime}\left(v^{\prime}, v^{\prime \prime}\right) & =1-\bar{y}(v), \quad \forall v \in V_{1}, \\
y^{\prime}(w) & =\bar{y}(w), \quad \forall w \in V_{2}, \\
y^{\prime}\left(v^{\prime}\right) & =\bar{y}(v), \quad \forall v \in V_{1}, \\
y^{\prime}\left(v^{\prime \prime}\right) & =1-\bar{y}(v), \quad \forall v \in V_{1} .
\end{aligned}
$$

We need the following two lemmas.

Lemma 3.2. There is a one to one correspondence between d-odd cycles in $G$ and g-odd cycles in $H$.

Proof. Let $C$ be a $d$-odd cycle of $G$, we build a cycle $C^{\prime}$ in $H$ as below. For every $\operatorname{arc}(u, v) \in A(C) \cap A_{1}$ we add $\left(u, v^{\prime}\right)$ to $C^{\prime}$, for every arc $(v, w) \in A(C) \cap A_{2}$ we add $\left(v^{\prime \prime}, w\right)$ to $C^{\prime}$. Finally for every node $v \in \tilde{C}$ we add the arc $\left(v^{\prime}, v^{\prime \prime}\right)$ to $C^{\prime}$. Notice that $\left|\tilde{C}^{\prime}\right|$ is even, thus $C^{\prime}$ is $g$-odd.

On the other hand if $D^{\prime}$ is a $g$-odd cycle of $H$ we obtain a cycle $D$ in $G$ by shrinking every arc $\left(v^{\prime}, v^{\prime \prime}\right)$ in $D$ into a single node. Clearly $D$ is $d$-odd.

Lemma 3.3. The vector $(\bar{x}, \bar{y})$ violates an inequality (3.1) if and only if $\left(x^{\prime}, y^{\prime}\right)$ violates an inequality (2.1).

Proof. Assume that there is a violated inequality (3.1), and let $C$ be the corresponding cycle of $G$. Let $C^{\prime}$ be the associated cycle in $H$. We have that $\left|\dot{C}^{\prime}\right|=|\dot{C}|$, $\left|\tilde{C}^{\prime}\right|=2|\tilde{C}|$, and $\sum_{v \in \hat{C}} \bar{y}(v)=\sum_{v \in \hat{C}^{\prime}} y^{\prime}(v)$.

For every node $v \in \tilde{C}$, we have $x^{\prime}\left(v^{\prime}, v^{\prime \prime}\right)=1-\bar{x}\left(\delta^{+}(v)\right)$. Thus

$$
\sum_{u \in \dot{C},(u, v) \in A(C)} \bar{x}(u, v)-\sum_{v \in \tilde{C}} \bar{x}\left(\delta^{+}(v) \backslash A(C)\right)+|\tilde{C}|=\sum_{(u, v) \in A\left(C^{\prime}\right)} x^{\prime}(u, v),
$$

and the lemma follows.

Therefore the separation problem for inequalities (3.1) reduces to the separation problem for inequalities (2.1) in the graph $H$. This can be done with the algorithm of $[4]$.

\subsection{Cut inequalities}

Let $S \subseteq V$ such that $S \cap V_{0} \neq \emptyset$. The following inequality is valid for $T \operatorname{LFLP}(G)$.

$$
x\left(\delta^{+}(S)\right)+y\left(V_{2} \cap S\right) \geq 1 .
$$


To see the validity, consider a $0-1$ vector $(\bar{x}, \bar{y})$ of $T L F L P(G)$. If $\bar{y}\left(V_{2} \cap S\right)=0$, then at least for one $\operatorname{arc}(u, v) \in \delta^{+}(S)$ we should have $\bar{x}(u, v)=1$.

Consider now the separation problem, and suppose that we are given a nonnegative vector $\left(x^{\prime}, y^{\prime}\right)$. We add an extra vertex $t$ and the $\operatorname{arcs}(w, t)$ for all $w \in V_{2}$. Then we define capacities $c$ for each arc as follows.

$$
\begin{aligned}
& c(u, v)=x^{\prime}(u, v) \quad \forall(u, v) \in A_{1} \cup A_{2}, \\
& c(w, t)=y^{\prime}(w) \quad \forall w \in V_{2} .
\end{aligned}
$$

Next we fix a node $u \in V_{0}$ and find a set of nodes $S$, with $u \in S, t \notin S$ that minimizes $c\left(\delta^{+}(S)\right)$. This can be done with a minimum cut algorithm in $O\left(|V|^{3}\right)$ time, see [3]. There is a violated inequality if and only if there is a set $\bar{S}$ with $c\left(\delta^{+}(\bar{S})\right)<1$. This procedure should be repeated for every node $u \in V_{0}$.

\subsection{Matching INEQUALities}

The following is an adaptation of a family of inequalities given in [2]. Suppose that $G_{1}$ admits a matching $M$ where for every node in $V_{1}$ there is an arc in $M$ incident to it, then the following inequality is valid for $\operatorname{TLF} L P(G)$.

$$
\sum_{(u, v) \in M} x(u, v)+\sum_{v \in V_{1}} y(v) \geq 2 .
$$

To see the validity, consider the case when from all the nodes in $V_{1}$ there is exactly one, $\bar{v}$, whose variable $y(\bar{v})$ takes the value one. Then we should have $x(\bar{u}, \bar{v})=1$, for $(\bar{u}, \bar{v}) \in M$.

The separation problem for a vector $(\bar{x}, \bar{y})$ can be solved by assigning the weight $\bar{x}(u, v)+\bar{y}(v)$ to each arc $(u, v) \in A_{1}$, then one should find a minimum weight matching that saturates every node in $V_{1}$.

\section{On the integrality of $P(G)$}

In this section we characterize the graphs for which $P(G)=T L F L P(G)$. Let $\bar{V}_{0}$ be the set of nodes $v \in V_{0}$ with $\left|\delta^{+}(v)\right|=1$. Let $\bar{V}_{1}$ be the set of nodes in $V_{1}$ that are adjacent to a node in $\bar{V}_{0}$. Clearly the variables associated with the nodes in $\bar{V}_{1}$ should be fixed, i.e., $y(v)=1$ for all $v \in \bar{V}_{1}$. Let $\bar{V}_{2}$ be the set of nodes in $V_{2}$ that are adjacent to a node $v \in \bar{V}_{1}$ with $\left|\delta^{+}(v)\right|=1$, we should have $y(w)=1$ for all $w \in \bar{V}_{2}$. Let us denote by $\bar{G}$ the subgraph induced by $V \backslash \bar{V}_{2}$ after removing all the $\operatorname{arcs}(u, v) \in A$ with $v \in \bar{V}_{1}$. We are going to prove the following.

Theorem 4.1. The polytope $P(G)$ is integral if and only if each cycle of $\bar{G}$ is d-even. 
First suppose that $\bar{G}$ contains a cycle $C$ that is $d$-odd. Let us define a fractional vector as follows:

- $\bar{y}(v)=1 / 2, \quad \forall v \in(\tilde{C} \cup \hat{C})$.

- $\bar{y}(v)=1, \quad \forall v \in \dot{C} \cap V_{1}$.

- $\bar{x}(a)=1 / 2, \quad \forall a \in A(C)$.

- $\bar{y}(v)=1, \quad \forall v \in V_{2} \backslash V(C)$.

- For every node $u \in V_{1} \backslash V(C)$, we look for an arc $(u, v) \in \delta^{+}(u)$, we set $\bar{x}(u, v)=\bar{y}(v)$. If $\left|\delta^{+}(u)\right| \geq 2$ and $\bar{y}(v)=1 / 2$, we pick another $\operatorname{arc}(u, w)$ and set $\bar{x}(u, w)=1 / 2$. Then we set $\bar{y}(u)=\bar{x}\left(\delta^{+}(u)\right)$.

- For every node $u \in V_{0} \backslash V(C)$, we look for an arc $(u, v) \in \delta^{+}(u)$, if $\bar{y}(v)=1$ we set $\bar{x}(u, v)=1$. If $\bar{y}(v)=1 / 2$ then there is another $\operatorname{arc}(u, w) \in \delta^{+}(u)$ with $\bar{y}(w)=1$ or $\bar{y}(w)=1 / 2$, then we set $\bar{x}(u, v)=\bar{x}(u, w)=1 / 2$.

- For every node $u \in V_{1} \cap \hat{C}$, we look for an $\operatorname{arc}(u, v) \in \delta^{+}(u)$ and set $\bar{x}(u, v)=$ $1 / 2$.

- For every other $\operatorname{arc} a$, we set $\bar{x}(a)=0$.

It is easy to see that $(\bar{x}, \bar{y}) \in P(G)$. Since

$$
\sum_{u \in \dot{C},(u, v) \in A(C)} \bar{x}(u, v)-\sum_{v \in \hat{C}} \bar{y}(v)-\sum_{v \in \tilde{C}} \bar{x}\left(\delta^{+}(v) \backslash A(C)\right)=\frac{|\dot{C}|}{2},
$$

we have that $(\bar{x}, \bar{y})$ violates an inequality (3.1) that is valid for $\operatorname{TLFLP}(G)$. So $P(G) \neq T \operatorname{LFLP}(G)$.

Now we assume that $\bar{G}$ has no $d$-odd cycle. First we need to introduce a related polytope studied in [4]. Let $H=(W, B)$ be a directed graph, and let $Q(H)$ be the polytope defined below.

$$
\begin{aligned}
& \sum_{(u, v) \in A} x(u, v)+y(u)=1 \quad \forall u \in W, \\
& x(u, v) \leq y(v) \quad \forall(u, v) \in B, \\
& 0 \leq y(v) \leq 1 \quad \forall v \in W, \\
& x(u, v) \geq 0 \quad \forall(u, v) \in B .
\end{aligned}
$$

In [4] we proved the following.

Theorem 4.2 [4]. If $H$ does not contain a g-odd cycle, then $Q(H)$ is integral.

In order to use Theorem 4.2 we have to transform $G$ to a directed graph $H$ such that $\bar{G}$ has no $d$-odd cycle if and only if $H$ has no $g$-odd cycle. This will be done in the following three steps.

- For every arc $a=(u, v)$ with $v \in \bar{V}_{1} \cup \bar{V}_{2}$, replace it by $\left(u, v_{a}\right)$, where $v_{a}$ is a new node.

- Split every node $v \in V_{1}$, replacing it by $v^{\prime}$ and $v^{\prime \prime}$, and adding an arc $\left(v^{\prime}, v^{\prime \prime}\right)$. Also every $\operatorname{arc}(u, v)$ is replaced by $\left(u, v^{\prime}\right)$, and every arc $(v, w)$ is replaced by $\left(v^{\prime \prime}, w\right)$. 
- For every node $w \in\left(V_{2} \backslash \bar{V}_{2}\right)$, add a node $w^{\prime}$ and an $\operatorname{arc}\left(w, w^{\prime}\right)$.

Let $H$ be this new graph. The goal here is to make a correspondence between some extreme point of $Q(H)$ and those of $P(G)$. If we consider any solution in $Q(H)$, then its restriction on the variables associated with $G$ may not belong to $P(G)$. To have the desired correspondence we study a face of $Q(H)$ defined as follows.

$$
\begin{aligned}
& y(u)=0, \text { for each node } u \in V_{0}, \\
& x\left(v^{\prime}, v^{\prime \prime}\right)=y\left(v^{\prime \prime}\right), \text { for each } \operatorname{arc}\left(v^{\prime}, v^{\prime \prime}\right) \text { with } v \in V_{1}, \\
& y\left(v^{\prime}\right)=1, \text { for each node } v^{\prime} \text { such that } v \in \bar{V}_{1},
\end{aligned}
$$

First notice that since $\bar{G}$ has no $d$-odd cycle, then $H$ has no $g$-odd cycle. Therefore, from Theorem 4.2, $Q(H)$ is an integral polytope. The polytope $P(G)$ is obtained from the face of $Q(H)$ defined above, by projecting the variables associated with extra nodes and extra arcs. Thus the proof of Theorem 4.1 is complete.

We can also state the following.

Theorem 4.3. The two level facility location problem is polynomially solvable for graphs $G$ such that $\bar{G}$ has no d-odd cycle.

In order to decide if $\bar{G}$ has a $d$-odd cycle, we start with $\bar{G}$ and split the nodes in $V_{1} \backslash \bar{V}_{1}$ as in Section 3.1. Let $\bar{H}$ be the new graph. We have seen that $\bar{G}$ has a $d$-odd cycle if and only if $\bar{H}$ has a $g$-odd cycle. In [4] we gave a polynomial algorithm to find a $g$-odd cycle in a graph, if there is any; this algorithm can be applied to $\bar{H}$.

\section{On the Facial STRUCture of TLFLP $(G)$}

Let $H$ be a bipartite graph $H=\left(U_{1} \cup U_{2}, B=U_{1} \times U_{2}\right)$. The uncapacitated facility location polytope of $H(U F L P(H))$ is the convex hull of all vectors satisfying

$$
\begin{aligned}
& \sum_{(u, v) \in B} x(u, v)=1, \quad \forall u \in U_{1} \\
& x(u, v) \leq y(v), \quad \forall(u, v) \in B \\
& x(u, v) \in\{0,1\}, \quad \forall(u, v) \in B \\
& y(v) \in\{0,1\}, \quad \forall v \in U_{2} .
\end{aligned}
$$

The facial structure of $U F L P(H)$ has been studied in [5-7,9] and others. In this section we extend some of these results to the two level case. Here we assume that $A_{1}=V_{0} \times V_{1}, A_{2}=V_{1} \times V_{2}$. First we study the dimension of $\operatorname{TLF} L P(G)$. Let $m=\left|V_{0}\right|, n=\left|V_{1}\right|$ and $p=\left|V_{2}\right|$. In what follows, we suppose that $n>1$ and $p>1$. 
Theorem 5.1. The dimension of $T L F L P(G)$ is $m n+p-m+n p$.

Proof. Since equations (1.1) and (1.3) are linearly independent, the dimension of $\operatorname{TLFLP}(G)$ is at most $m n+p-m+n p$.

Now suppose that $T L F L P(G) \subseteq\{(x, y) \mid a x+b y=\alpha\}$. Adding multiples of equations (1.3), we may assume that $b(v)=0$ for all $v \in V_{1}$. Also adding multiples of equations (1.1) we may assume that $a\left(u, v_{0}\right)=0$ for a fixed node $v_{0} \in V_{1}$ and all $u \in V_{0}$. Let $\left(x^{1}, y^{1}\right)$ be defined as follows.

$$
\begin{aligned}
& x^{1}\left(u, v_{0}\right)=1, \quad \forall u \in V_{0}, \\
& x^{1}\left(v_{0}, w_{0}\right)=1, \text { for a fixed node } w_{0} \in V_{2}, \\
& y^{1}\left(v_{0}\right)=y^{1}\left(w_{0}\right)=1, \\
& x^{1}(u, v)=0 \text { otherwise, } \\
& y^{1}(v)=0 \text { otherwise. }
\end{aligned}
$$

We have that $a x^{1}+b y^{1}=\alpha$. Now for $w \in V_{2}, w \neq w_{0}$, let $\left(x^{2}, y^{2}\right)$ be the vector obtained from $\left(x^{1}, y^{1}\right)$ by setting $y(w)=1$. Since $a x^{2}+b y^{2}=\alpha$, we have $b(w)=0$, for $w \in V_{2}, w \neq w_{0}$. Since $\left(x^{1}, y^{1}\right)$ can also be built with a node different from $w_{0}$, we also have $b\left(w_{0}\right)=0$.

Now starting from $\left(x^{2}, y^{2}\right)$ let $\left(x^{3}, y^{3}\right)$ be the vector obtained by setting $x\left(v_{0}, w_{0}\right)=0$ and $x\left(v_{0}, w\right)=1$. Since $a x^{3}+b y^{3}=\alpha$, we have $a\left(v_{0}, w_{0}\right)=a\left(v_{0}, w\right)$ for $w \in V_{2}, w \neq w_{0}$. Since the same construction can be done with a node different from $v_{0}$, we have that $a\left(v, w_{0}\right)=a(v, w)$ for $w \in V_{2}, w \neq w_{0}$, for any node $v \in V_{1}$.

Now starting from $\left(x^{1}, y^{1}\right)$, set $x(v, w)=1, y(v)=1, y(w)=1$, for a node $v \in V_{1}, v \neq v_{0}$, and a node $w \in V_{2}, w \neq w_{0}$. Denote by $\left(x^{4}, y^{4}\right)$ this new vector, we have that $a x^{4}+b y^{4}=\alpha$, therefore $a(v, w)=0$. Since the same construction can be done with nodes different from $v_{0}$ and $w_{0}$, we have that $a(v, w)=0$ for all $v \in V_{1}$, and all $w \in V_{2}$.

Finally, starting from $\left(x^{1}, y^{1}\right)$, set $x(u, v)=1, y(v)=1$, for a node $u \in V_{0}$, $v \in V_{1}, v \neq v_{0}, x\left(v, w_{0}\right)=1, x\left(u, v_{0}\right)=0$. Let $\left(x^{5}, y^{5}\right)$ be this new vector. Since $a x^{5}+b y^{5}=\alpha$, we have that $a(u, v)=a\left(u, v_{0}\right)=0$. Since $u$ and $v$ can be chosen arbitrarily, we have $a(u, v)=0$ for all $u \in V_{0}$ and all $v \in V_{1}$.

This shows that there is no equation linearly independent from (1.1) and (1.3) that is satisfied by all vectors in $\operatorname{TLFLP}(G)$, thus its dimension is $m n+p-m+n p$ and its affine hull is defined by (1.1) and (1.3).

Recall that $G_{1}$ is the subgraph induced by $V_{0} \cup V_{1}$, and $G_{2}$ is the subgraph induced by $V_{1} \cup V_{2}$.

\subsection{Facets associated with $\operatorname{UFL}\left(G_{1}\right)$}

The following theorem shows that all facets of $U F L P\left(G_{1}\right)$ give facets of $\operatorname{TLFLP}(G)$. 
Theorem 5.2. If

$$
\sum_{(u, v) \in A_{1}} a(u, v) x(u, v)+\sum_{v \in V_{1}} b(v) y(v) \leq \alpha
$$

defines a facet of $U F L P\left(G_{1}\right)$, then it also defines a facet of $T L F L P(G)$.

Proof. Clearly this inequality is valid for $\operatorname{TLFLP}(G)$. Let $F$ be the facet of $U F L P\left(G_{1}\right)$ defined by (5.5), and let $F^{\prime}$ be the face of $T L F L P(G)$ defined by $(5.5)$. Let us assume that

$$
F^{\prime} \subset\{(x, y) \mid c x+\mathrm{d} y=\beta\},
$$

where $c x+\mathrm{d} y \leq \beta$ is valid for $\operatorname{TLFLP}(G)$.

Let $v \in V_{1}$, the inequality $y(v) \geq 0$ does not define a facet of $U F L P\left(G_{1}\right)$, see [9]. Thus there is a $0-1$ vector $\left(x^{v}, y^{v}\right)$ in $F$ with $y(v)=1$. For each node $v^{\prime} \in V_{1}$ with $y^{v}\left(v^{\prime}\right)=1$, we define $x^{v}\left(v^{\prime}, w_{0}\right)=1, y^{v}\left(w_{0}\right)=1$, where $w_{0}$ is a fixed node in $V_{2}$. Then we set $x^{v}(u, w)=0$ for every other $\operatorname{arc}(u, w) \in A_{2}$, and $y^{v}(w)=0$ for every other node $w \in V_{2}$. This new vector $\left(\bar{x}^{v}, \bar{y}^{v}\right)$ belongs to $F^{\prime}$.

Starting from $\left(\bar{x}^{v}, \bar{y}^{v}\right)$, pick a node $w \in V_{2}, w \neq w_{0}$, and set $y(w)=1$. Since this new vector also belongs to $F^{\prime}$, we have $d(w)=0$. Since the node $w_{0}$ was chosen arbitrarily, and the same construction can be done with a different node, we have that $d(w)=0$ for all $w \in V_{2}$.

Now starting again from $\left(\bar{x}^{v}, \bar{y}^{v}\right)$, set $x\left(v, w_{0}\right)=0$ and $x(v, w)=y(w)=1$, where $w \in V_{2}, w \neq w_{0}$. Since this new vector also belongs to $F^{\prime}$, we have $c(v, w)=$ $c\left(v, w_{0}\right)$. Thus we have that $c(v, w)=\lambda_{v}$, for all $w \in V_{2}$. We repeat this for all $v \in V$ and we have $c(v, w)=\lambda_{v}$, for all $v \in V_{1}$ and $w \in V_{2}$.

Thus $c x+\mathrm{d} y \leq \beta$ can be written as

$$
\sum_{(u, v) \in A_{1}} c(u, v) x(u, v)+\sum_{v \in V_{1}}\left(d(v)+\lambda_{v}\right) y(v) \leq \beta .
$$

Thus (5.6) defines a face of $\operatorname{UF} L P\left(G_{1}\right)$. Since (5.5) defines a facet of $U F L P\left(G_{1}\right)$, then (5.6) can be obtained by taking a positive multiple of (5.5) and adding multiples of equations (1.1). This shows that (5.5) defines a facet of $T L F L P(G)$.

Corollary 5.3. The following inequalities define facets of $\operatorname{TLFLP}(G)$.

$$
\begin{aligned}
& x(u, v) \leq y(v), \quad \forall(u, v) \in A_{1} \\
& x(u, v) \geq 0, \quad \forall(u, v) \in A_{1} \\
& y(v) \leq 1, \quad \forall v \in V_{1} .
\end{aligned}
$$

Proof. These inequalities define facets of $\operatorname{UFLP}\left(G_{1}\right)$, see [9].

Corollary 5.4. Inequalities (3.3) define facets of $T L F L P(G)$.

Proof. It follows from Theorems 6 and 8 in [2] that (3.3) define facets of $U F L P\left(G_{1}\right)$. 


\subsection{Facets associated with $U F L P\left(G_{2}\right)$}

Let $U F L P^{\prime}\left(G_{2}\right)$ the relaxation of $\operatorname{UFLP}\left(G_{2}\right)$ obtained by replacing equations (5.1) by inequalities, that is the convex hull of the solutions of the system below

$$
\begin{aligned}
& \sum_{(v, w) \in A_{2}} x(v, w) \leq 1, \quad \forall v \in V_{1}, \\
& x(v, w) \leq y(w), \quad \forall(v, w) \in A_{2}, \\
& x(v, w) \in\{0,1\}, \quad \forall(v, w) \in A_{2}, \\
& y(w) \in\{0,1\}, \quad \forall w \in V_{2} .
\end{aligned}
$$

Notice that any facet of $\operatorname{UFLP}\left(G_{2}\right)$ is contained in a facet of $\operatorname{UFLP}\left(G_{2}\right)$. In [9], it has been seen that the polytope $U F L P^{\prime}\left(G_{2}\right)$ corresponds to a stable set polytope. Define $\bar{y}(w)=1-y(w)$ for each node $w \in V_{2}$, then the system above may be rewritten as follows:

$$
\begin{aligned}
& \sum_{(v, w) \in A_{2}} x(v, w) \leq 1, \quad \forall v \in V_{1}, \\
& x(v, w)+\bar{y}(w) \leq 1, \quad \forall(v, w) \in A_{2}, \\
& x(v, w) \in\{0,1\}, \quad \forall(v, w) \in A_{2}, \\
& y(w) \in\{0,1\}, \quad \forall w \in V_{2} .
\end{aligned}
$$

Let $A$ be the 0-1 matrix associated with the constraints (5.7) and (5.8). An undirected graph $H$, called the intersection graph of $A$, is built containing a node for each variable $x(v, w) \in A_{2}$ and a node for each variable $\bar{y}$. For any two variables $\left(v, w_{1}\right)$ and $\left(v, w_{2}\right)$ where $\left(v, w_{1}\right)$ and $\left(v, w_{2}\right)$ are arcs of $G_{2}$, we have an edge between the corresponding nodes; thus there is a maximal clique $K(v)$ for each node $v \in V_{1}$. Also for every variable $x(v, w)$ there is an edge between the node associated with $x(v, w)$ and the node associated with $\bar{y}(w)$. We have the following well known result.

Theorem 5.5. [9] The convex hull of the feasible solutions of (5.7)-(5.10) is exactly the stable set polytope of $H$.

To establish the theorem that shows that all facets of $U F L P^{\prime}\left(G_{2}\right)$ give facets of $\operatorname{TLF} L P(G)$, we need the lemma below.

Lemma 5.6. If $a x+b y \leq \alpha$ defines a facet $F$ of $U F L P^{\prime}\left(G_{2}\right)$, then there is a $0-1$ vector $(\bar{x}, \bar{y}) \in F$ such that

$$
\sum_{w} \bar{x}(v, w)=1, \text { for all } v \in V_{1}
$$

Proof. Here we follow the approach of [9] associating a stable set polytope with $U F L P^{\prime}\left(G_{2}\right)$. Let $S S P(H)$ the stable set polytope of $H$. The facets of $U F L P^{\prime}\left(G_{2}\right)$ 
correspond to the facets of $S S P(H)$. For a "trivial" facet of $S S P(H)$ defined by $x(s) \geq 0$, we just pick a node different from $s$ in every maximal clique of $H$.

Now we have to consider a nontrivial facet of $S S P(H)$ defined by $a x \leq \alpha$. We have that $a \geq 0, \alpha>0$. Let $x^{W}$ be the incidence vector of a stable set of $H$ such that $a x^{W}=\alpha$. We may assume that $W$ is a maximal stable set. Since $\sum_{w \in V_{2}} x(w) \leq\left|V_{2}\right|$ does not define a facet, we may assume that $x^{W}\left(w_{0}\right)=0$ for some node $w_{0} \in V_{2}$. So for every clique $K(v)$ we can try to add to $W$ the node associated with $\left(v, w_{0}\right)$, if this is not possible it is because another node in $K(v)$ is already in $W$. Thus we may assume that for each $v \in V_{1}$ there is a node in $K(v)$ that belongs to $W$.

Theorem 5.7. If

$$
\sum_{(v, w) \in A_{2}} a(v, w) x(v, w)+\sum_{w \in V_{2}} b(w) y(w) \leq \alpha
$$

defines a facet of $U F L P^{\prime}\left(G_{2}\right)$, then it also defines a facet of $\operatorname{TLFLP}(G)$.

Proof. Clearly (5.11) is valid for $T L F L P(G)$. Let $F$ be the facet of $U F L P^{\prime}\left(G_{2}\right)$ defined by (5.11), and let $F^{\prime}$ be the face of $T L F L P(G)$ defined by (5.11). Let us assume that

$$
F^{\prime} \subset\{(x, y) \mid c x+\mathrm{d} y=\beta\}
$$

where $c x+\mathrm{d} y \leq \beta$ is valid for $\operatorname{TLFLP}(G)$.

Let $(\bar{x}, \bar{y})$ be the vector defined in Lemma 5.6. Starting from this vector, fix a node $v_{0} \in V_{1}$ and set $x\left(u, v_{0}\right)=1$ for all $u \in V_{0}$. Set $x(u, v)=0$ for every other $\operatorname{arc}(u, v) \in G_{1}$, and $y(v)=1$ for all $v \in V_{1}$. Denote by $(\tilde{x}, \tilde{y})$ this new vector. We have that $(\tilde{x}, \tilde{y}) \in F^{\prime}$. Starting from $(\tilde{x}, \tilde{y})$, pick a node $v_{1} \in V_{1}, v_{1} \neq v_{0}$, a node $u_{0} \in V_{0}$ and set $x\left(u_{0}, v_{0}\right)=0, x\left(u_{0}, v_{1}\right)=1$. Denote by $(\hat{x}, \hat{y})$ this last vector. We have that $(\hat{x}, \hat{y}) \in F^{\prime}$, thus $c\left(u_{0}, v_{0}\right)=c\left(u_{0}, v_{1}\right)$. Since $v_{1}$ was chosen arbitrarily, we have $c\left(u_{0}, v_{0}\right)=c\left(u_{0}, v\right)$ for all $v \in V_{1}$. Since $u_{0}$ was chosen arbitrarily, we have $c(u, v)=\lambda_{u}$ for all $u \in V_{0}$ and all $v \in V_{1}$.

Therefore by adding multiples of equations (1.1) to $c x+\mathrm{d} y \leq \beta$, we obtain a new inequality

$$
c^{\prime} x+d^{\prime} y \leq \beta^{\prime}
$$

with $c^{\prime}(u, v)=0$ for all $u \in V_{0}$ and all $v \in V_{1}$. By adding multiples of equations (1.3), we may assume that $d^{\prime}(v)=0$ for all $v \in V_{1}$.

Thus (5.12) defines a face of $U F L P^{\prime}\left(G_{2}\right)$. Since this is a full dimensional polytope, we have that (5.12) is a positive multiple of (5.11). Therefore (5.11) defines a facet of $T L F L P(G)$. 
Corollary 5.8. The following inequalities define facets of $T L F L P(G)$.

$$
\begin{aligned}
& y(u)=\sum_{(u, v) \in A_{2}} x(u, v) \leq 1 \quad \forall u \in V_{1} \\
& x(u, v) \leq y(v), \quad \forall(u, v) \in A_{2} \\
& x(u, v) \geq 0, \quad \forall(u, v) \in A_{2} \\
& y(v) \leq 1, \quad \forall v \in V_{2} .
\end{aligned}
$$

Proof. These inequalities define facets of $U F L P^{\prime}\left(G_{2}\right)$, see [9].

Acknowledgements. We are grateful to an anonymous referee for its careful reading of the manuscript, and for pointing out an error in the statement of Theorem 4.1.

\section{REFERENCES}

[1] K. Aardal, F.A. Chudak and D.B. Shmoys, A 3-approximation algorithm for the $k$-level uncapacitated facility location problem. Inform. Process. Lett. 72 (1999) 161-167.

[2] K. Aardal, M. Labbé, J. Leung and M. Queyranne, On the two-level uncapacitated facility location problem. INFORMS J. Comput. 8 (1996) 289-301.

[3] R.K. Ahuja, T.L. Magnanti and J.B. Orlin, Network flows, Theory, algorithms, and applications, Prentice Hall Inc., Englewood Cliffs, NJ (1993).

[4] M. Baïou and F. Barahona, On the integrality of some facility location polytopes. SIAM J. Discrete Math. 23 (2009) 665-679.

[5] L. Cánovas, M. Landete and A. Marín, On the facets of the simple plant location packing polytope. Discrete Appl. Math. 124 (2002) 27-53.

[6] D.C. Cho, E.L. Johnson, M. Padberg and M.R. Rao, On the uncapacitated plant location problem. I. Valid inequalities and facets. Math. Oper. Res. 8 (1983) 579-589.

[7] D.C. Cho, M.W. Padberg and M.R. Rao, On the uncapacitated plant location problem II. Facets and lifting theorems. Math. Oper. Res. 8 (1983) 590-612.

[8] V. Chvátal, Edmonds polytopes and a hierarchy of combinatorial problems. Discrete Math. 4 (1973) 305-337.

[9] G. Cornuejols and J.-M. Thizy, Some facets of the simple plant location polytope. Math. Progr. 23 (1982) 50-74.

[10] E. Kantor and D. Peleg, Approximate hierarchical facility location and applications to the bounded depth Steiner tree and range assignment problems. J. Discrete Algorithms 7 (2009) $341-362$. 